\title{
Effect of the Operating Parameters on the Magnetic Filter Constructed from the Granular Beds
}

\author{
Zehra Yildiz \\ Department of Energy Systems Engineering, Technology Faculty, Mersin University, Mersin, Turkey \\ Email address: \\ zyildiz@mersin.edu.tr \\ To cite this article: \\ Zehra Yildiz. Effect of the Operating Parameters on the Magnetic Filter Constructed from the Granular Beds. American Journal of Chemical \\ Engineering. Vol. 5, No. 6, 2017, pp. 154-157. doi: 10.11648/j.ajche.20170506.16
}

Received: July 27, 2017; Accepted: August 28, 2017; Published: November 28, 2017

\begin{abstract}
In this article, magnetizing properties of the packed beds constructed from ferromagnetic spheres and chips were investigated experimentally in order to determine some effect of the separation efficiency of the magnetized granular beds. Effects of the external magnetic field intensity, filter length and cycle number on the effectiveness of electromagnetic filtration of both solutions were also investigated. It was found that the capturing capacity of the processed particles in the filter increases with increasing external magnetic field intensity, filter length and cycle number until the filter reached to its saturation at this point.
\end{abstract}

Keywords: Magnetic Granules, Magnetized Packed Bed, Electromagnetic Filtration

\section{Introduction}

In the recent years, the water consumption for various practical applications like heating, cooling, energy producing, etc., has been incredibly increased with the developments of the various industrial branches and the rise in the world population. It is clear that water in these applications must have some certain qualities to meet these requirements. Some of the known impurities found in water used in the industry are mostly $\mathrm{Fe}$ and its various compounds, and the rest are $\mathrm{Ni}$, $\mathrm{Cu}, \mathrm{Cr}, \mathrm{Co}, \mathrm{Al}, \mathrm{Zn}, \mathrm{Ti}, \mathrm{Zr}, \mathrm{Mn}$ and some alloys. Although some of these impurities are found in waters naturally, they are mostly produced during the processes as a result of corrosion and other factors. Even though amount of them are very small, they can cause serious problems asmentioned in the literature [1-3]. Essentially, various separation methods can be applied depending on the characteristics of the solids and the ratio of the solid/liquid in the suspension. However, if the particle concentration is around $10^{-6}-10^{-8} \mathrm{ppm}$ and their sizes are lower than $1 \mu \mathrm{m}$, the conventional methods fail to supply a high degree of separation. Therefore, this inadequacy of conventional filters is remedied by the use of electromagnetic filters which have become very popular recently and successfully used for the separation of heavy metal ions, phosphates, and corrosion products like rusts in mining, glass, ceramic, oil, power and nuclear power generation industries [3-6]. The matrices of an electromagnetic filter are composed of the ferromagnetic packed beds that can be easily magnetized under an external magnetic field. The packing elements are usually spherical balls, steel wools, metal rods and wires [1,7-10]. The local high gradient fields are formed around these packing elements through the effect of the external magnetic field. Purification of a liquid or a gas is then accomplished by passing the suspension and holding the micron sized magnetic dispersed particles in these areas. Since ferromagnetic packed beds can withstand high temperatures, corrosive and radioactive mediums, mechanical and hydrodynamic effects, they can be effectively used in the separation processes of many industrial brunches [3-5, 1112]. Recently, new application areas of electromagnetic filters in medicine, [3, 9-12], chemical technology and nanofluid systems $[10,13]$ have been rapidly increased. The performance of these filter equipments depends on the magnetic, hydrodynamic, geometric and rheological parameters of the system to be cleaned [13-17]. In order to suggest a general theory for this process, effects of all these parameters must be known, however, there are not many studies carried out to enlighten these points. For this reason, the most important problems to be solved are the determination of the kinetics of magnetic filter and the relationship between the parameters affecting the filtration 
process. On the other hand, the matrices of magnetic filters are generally constructed from ferromagnetic spheres [3-4, 7$8,14]$. Considering a filter having $1 \mathrm{~m}$ length to be used for practical purposes, it is clear that amount of spherical packing bed elements will be very high resulting in high operational costs. Moreover, if the spheres are required to be plated with special anti-corrosive material, then the filter will become more expensive and disadvantageous. For this reason, use of waste steel chips and pieces of wires, and also recycling the filtration process may reduce the costs. In this case, magnetizing properties of filter matrices and process parameters must be investigated, and their effects on the filter performance must be determined.

\section{Experimental Method}

For the experimental studies in this work the packed bed is placed into a rust proof case with dimensions of $0.04 \mathrm{~m}$ $\mathrm{x} 0.03 \mathrm{~m} \times 0.1 \mathrm{~m}$. The electromagnetic filter used in the experimental studies is consisted of non magnetic filter body and rust proof steel balls, rods and chips as the filter elements. As the external magnetizing medium, multipurpose electromagnetic magnetizing equipment (AC/DC, 0-220 V, and 0-20 A) has been used. The experimental studies have been carried out by placing an electromagnetic filter into this equipment which has a $48 \mathrm{~mm}$ diameter. Magnetic field intensity (B) was within the range of $0-1.5 \mathrm{~T}$. The local magnetic field intensities at 14 different points along the bed are measured and evaluated using GM 05 Gaussmeter (Hirst Magnetic Instruments Ltd.).

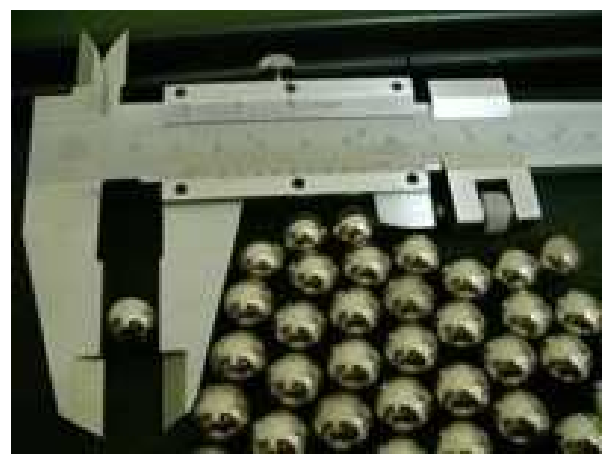

(a)

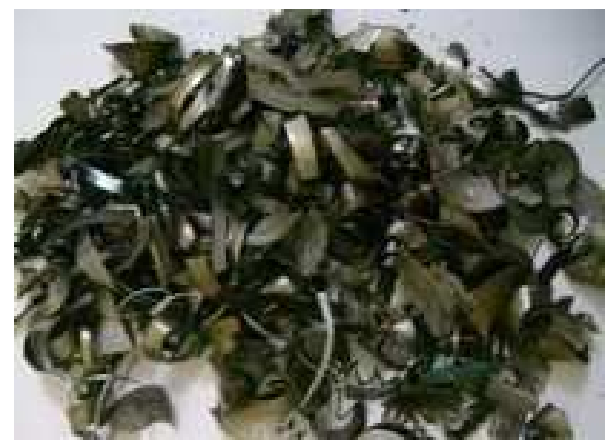

(b)

Figure 1. Types of ferromagnetic packing elements a) Ferromagnetic spheres b) Ferromagnetic chips.
Ferromagnetic spheres with diameters of $6.28 \mathrm{~mm}$ are used as a bed element. In order to keep the ferromagnetic bed more economical, on the other hand, ferromagnetic metal chips were also used as mentioned previously. Types of the bed elements used in the present work are shown in Fig.1.

The most important characteristics of the electromagnetic filters that makes them more useful and popular compared to classical filters was the separation of nano particles from the carrier medium with a very high efficiency. For this reason, Scanning Electron Microscope (SEM) analysis was necessarily made in order to determine mean particle sizes of the rust particles that form the suspension. From the measurements taken from the 11 different points of sample, particle size distribution for the samples has been determined in terms of micrometers $(\mu \mathrm{m})$. As can be seen from Fig.1, the mean particle sizes vary between $0.2-2(\mu \mathrm{m})$. However, in this work, the mean particle size was taken as $2(\mu \mathrm{m})$ for the calculation purposes.

The feed solution to be filtered was prepared by mixing the tap water and the rust particles, the amount of $\mathrm{Fe}$ were determined from the analysis taken using Atomic Absorption Spectrometer (AAS) equipments.

Experiments were carried for various conditions, such as external magnetic field intensity of 0,22-0.35 T, filter length of 1-10 cm, cycle number of 1-7. In order to prevent the coalescence of the rust particles, a continuous mixing was applied. After determining the total $\mathrm{Fe}$ amount, the cleaning performance of the filtration process, which is also called as quality factor $(\psi)$, was determined using the following equation:

$$
\psi=\lambda \frac{C_{i}-C_{o}}{C_{i}}
$$

where, $\lambda$, the ferromagnetic fraction of the mixture, $C_{i}$ and $C_{o}$ are the total $\mathrm{Fe}$ amount at the inlet and outlet respectively $(\mathrm{mg} / \mathrm{L})$.

\section{Results and Discussions}

\subsection{Filter Length}

The experimental studies were carried out using the conditions described as above with the value of external magnetic field intensity as $0.32 \mathrm{~T}$ and the filter length as 1-10 $\mathrm{cm}$ without any cycling. As can be seen from Fig. 2, if the external magnetic field intensity was $0.32 \mathrm{~T}$, the filter efficiency increased from 21 to $83 \%$ for ferromagnetic spheres and from 8 to $43 \%$ for chips. Capturing ratio was found to be maximum in filters constructed from ferromagnetic spheres, while the lowest was found from metal chips.

However, regardless of the type of the bed element, it is clear that after the length of $5 \mathrm{~cm}$, the variation in the filter efficiency was negligible as the filter reached to its saturation at this point. Because of this, it is very important the determination of the magnetic saturation in both the filtration economics and the cleaning performance. Amount of the filter package elements increase the filter length. It is more 
expensive amount of the filter package elements on the electromagnetic filtration cost. Actually, the magnetic filter is about a few meters and consist of hundreds kilos filter elements in the many industrial branches.

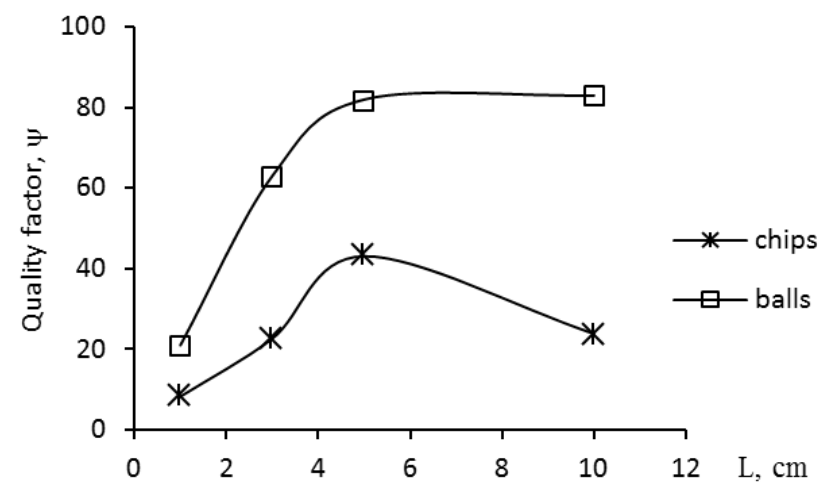

Figure 2. Variation of the quality factor with the filter length in the electromagnetic filter $V_{f}=0.15 \mathrm{~m} / \mathrm{s}, C_{i}=50 \mathrm{ppm}, B=0.32 T$.

\subsection{Magnetic Field Intensity}

For the experimental purposes the external magnetic field intensity was changed within the range of $0.22-0.35 \mathrm{~T}$. As noted from Fig. 3, the increase of the intensity values from 0.22 to $0.32 \mathrm{~T}$ resulted in an increased efficiency of $43-83 \%$ for ballsand $25-52 \%$ for chips. However up to the value of $0.32 \mathrm{~T}$, this increase was important and then it was negligible. On the other hand, at the value of $0.35 \mathrm{~T}$, the quality factor decreased for all cases since the filter reached to its saturation point. However, the electromagnetic filter that is constructed the metal chips as metal waste is cheap. On the other hand, the electromagnetic filter is poor magnetically. The quality factor is less than the electromagnetic filter that is constructed the balls. The balls are most efficiently in the magnetic filter package elements according to the cleaning filter performance.

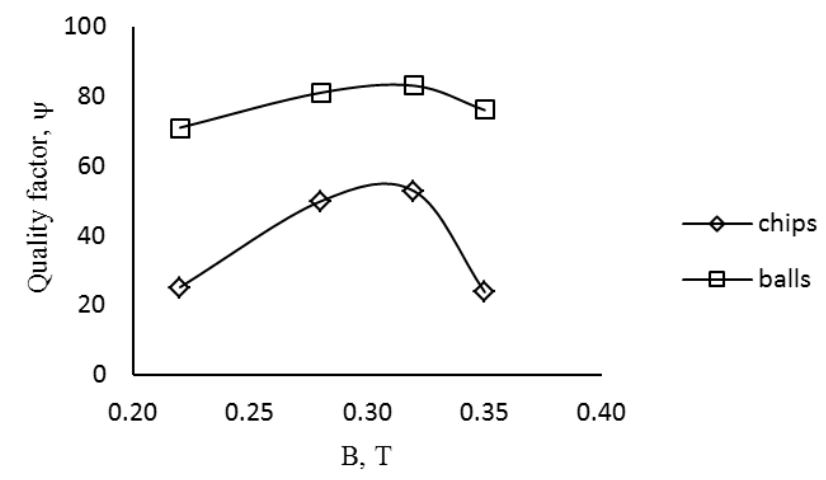

Figure 3. Variation of the quality factor with the magnetic field intensity in the electromagnetic filter $L=0,1 \mathrm{~m}, C_{i}=50 \mathrm{ppm}, V_{f}=0,15 \mathrm{~m} / \mathrm{s}$.

\subsection{Number of Cycles}

The amount of the cycle number was ached between 1-7 for $0.32 \mathrm{~T}$ and it was recorded that for the filter lengths of $3 \mathrm{~cm}$, a noticeable increase was seen up to the cycle number of 5 (fig. 4). Increasing the cycle number did not lead to any recordable variation in the efficiency at this external magnetic field intensity value. Therefore, the use of shorter filter by increasing the amount of the cycles can provide lowering the initial costs of the processes and supplying more economical separations.

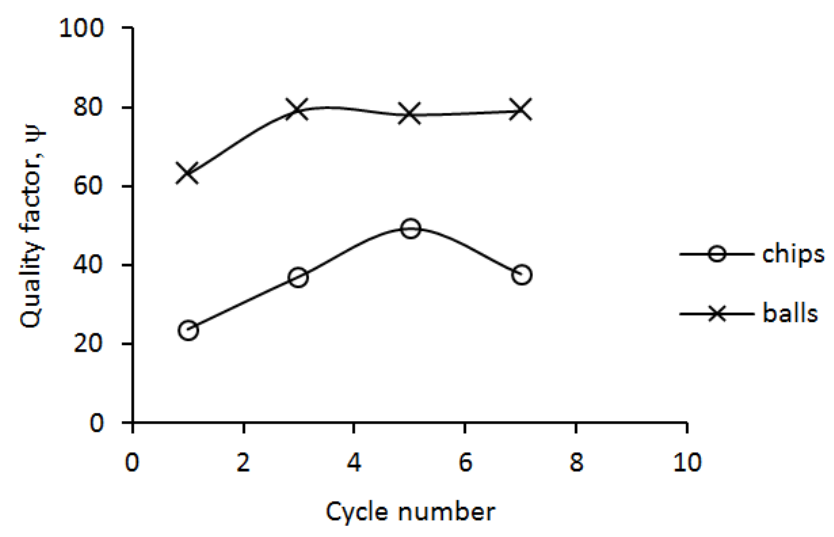

Figure 4. Variation of the quality factor with the number of cycles used in the electromagnetic filter $L=0.03 \mathrm{~m}, C_{i}=50 \mathrm{ppm}, V_{f}=0.15 \mathrm{~m} / \mathrm{s}, B=0.32 \mathrm{~T}$.

In addition, Figure 5 shows clearly that the quality factor of the electromagnetic filter that is constructed the balls increase the number cycles. Respectively, it is a, b and c, unfiltered corrosion products (rust) suspension, the number cycle of electromagnetic filtered 3 times and the number cycle of electromagnetic filtered 5 times. It is resulted that cleaning performance of the electromagnetic filter in five times cycle filtered suspension is 2 multiple better than unfiltered.

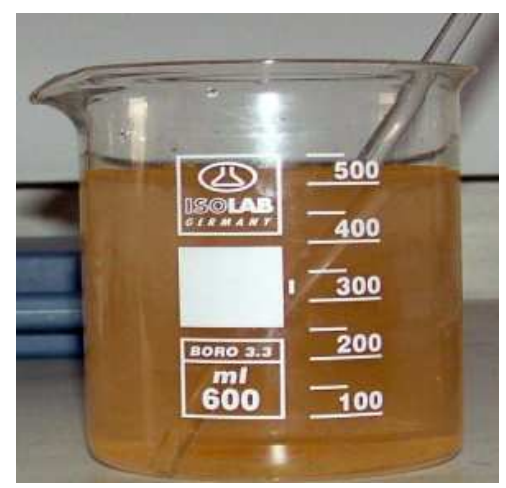

(a)

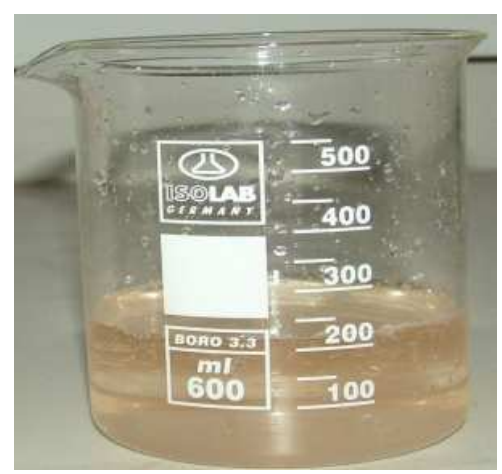

(b) 


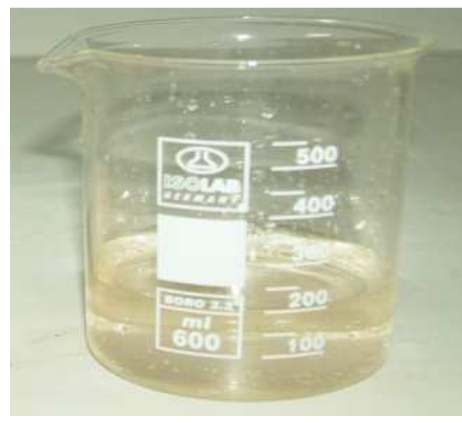

(c)

Figure 5. Cleaning performancewith the number of cycles used in the electromagnetic filter.

\section{Conclusions}

From the experimental studies of the packed beds constructed from the ferromagnetic elements, the followings can be withdrawn:

The porous media constructed from the ferromagnetic elements that can be easily magnetized in an external magnetic field have high magnetic characteristics. Ferromagnetic balls showed the strongest magnetizing characteristics under the external magnetic field intensity values used, while chips the lowest.

Increasing the packing factor, the characteristics mentioned above improved. From the practical point of view, on the other hand, homogeneous balls are more advantageous. From the results from all experiments, the highest cleaning efficiency was obtained by using ferromagnetic spheres.

Although their relatively low magnetizing characteristics, the use of the packed beds with ferromagnetic chips are more economical in the practical applications. However, the cleaning efficiency is quite low by using ferromagnetic chips compared to those by using spheres as mentioned above. Even the use of a cycle in the filter process could not provide such high degree of separation from the carrier medium. Although the filters constructed from metal chips are cheaper, maximum degree of separation were about $55 \%$ because of the very low porous structure of the medium. However, if very high degree of separation is not required, then these sort of packing elements could easily be used. This way, reusing of the waste material will contribute to cleaner environment and economic situations of the world.

\section{References}

[1] J. Svoboda, "Magnetic techniques for the treatment of materials", Kluwer AcdemicPublishers, USA, 2004.

[2] A. V. Sandulyak, "Magnetic filtration of liquids and gases", Ximiya, Moscow, 1988.
[3] T. Abbasov, "Electromagnetic filtration processes, Theory, Application and Construction" Seckin, Ankara, 2002.

[4] B. E. Kashevskii, I. V. Prokhorov, "Magnetophoretic potential of a chain of ferromagnetic balls in a homogeneous field", Journal of Engineering Physics and Thermophysics, 76, 775$82,2003$.

[5] A. Sandulayak, A. Sandulayak, V. Ershova, M. Polismakova, D. Sandulayak, "Use of the magnetic test- filter for magnetic control of ferroimpurities of fuels, oils, and other liquids", Journal of Magnetism and Magnetic Materials, 426, 714-720, 2017.

[6] J. Y. Hristov, "Fluidization of ferromagnetic particles in a magnetic field Part 1: the effect of field line orientation on bed stability”, Powder Technology, 87, 59-66, 1996.

[7] C. Moyer, M. Natenapit, S. Arajs, "Particle capture by an assemblage of spheres in HGMS", J. Appl. Phys., 55, 2589$2592,1984$.

[8] F. J. Friedlaender, R. Gerber, W. Kurz, R. R. Birrs, "Particle motion near and capture on single spheres in HGMS", IEEE Trans. Magn., MAG-17, 2801-4, 1981.

[9] L. Sun, M. Zborowski, L. R. Moore, J. J. Chalmers, "Continuous, flowthroughimmunomagnetic cell sorting in aquadrupole field", Cytometry, 33, 469-475, 1998.

[10] O. Rotaiu, N. J. C. Strachan, "Modelling magnetic carrier particle targeting in the tumor microvasculature for cancer treatment", Journal of Magnetism and Magnetic Materials, 293, 639-646, 2005.

[11] L. E. Udreal, N. J. C. Strachan, V. B. Badescu, O. Rotariu, 'An in vitro study of magnetic particle targeting in small blood vessels", Phys. Med. Biol., 51, 4869-4881, 2006.

[12] C. Alexiou, D. Dieh, P. Henninger, H. Iro, R. Röckelein, W. Schmidt, H. Weber, "A high field gradient magnet for magnetic drug targeting", IEEE Trans. Magn. Superconductivity, 16, 1527-1530, 2006.

[13] T. Abbasov, V. Gögebakan, T. Karadağ, "Particle capture modeling for anaxial magnetic fitler with abounded nonNewtonian flow fiel", Powder Technology, 29, 1223-1228, 2016.

[14] M. Yuceer, Z. Yildiz, T. Abbasov, Evaluation of Electromagnetic Filtration Efficiency Using LS-SVM', Physicochemical Problems of Mineral Processing, 51, 173-180, 2015

[15] I. Iluita, F. Larachi, "Theory of tricle-bed magnetohydrodynamics under magnetic field gradients", AIChe J., 49, 1525-1532, 2003.

[16] E. P. Furlani, Y. Sahoo, "Analytical model for the magnetic field and force in a magnetophoretic microsystem", J. Phys. D: Appl. Phys., 9, 39, 1724-1732, 2006.

[17] N. Hirota, T. Ando, T. Takano, H. Okada, "In-situ observation of particles deposition process on a ferromagnetic filter during high-gradient magnetic separation process", Journal of Magnetism and Magnetic Materials, 427, 269-299, 2017. 